\title{
Article \\ Binary-Encounter Model for Direct Ionization of Molecules by Positron-Impact
}

\author{
Małgorzata Franz ${ }^{1, *,+(\mathbb{D})}$, Katarzyna Wiciak-Pawłowska ${ }^{1, *,+} \mathbb{D}$ and Jan Franz and $^{1, *,+}(\mathbb{D})$ \\ 1 Faculty of Applied Physics and Mathematics, Gdańsk University of Technology, 80-233 Gdańsk, Poland \\ 2 Advanced Materials Center, Gdańsk University of Technology, 80-233 Gdańsk, Poland \\ * Correspondence: malobaro@pg.edu.pl (M.F.); katarzyna.pawlowska@pg.edu.pl (K.W.-P.); \\ janfranz@pg.edu.pl (J.F.) \\ + These authors contributed equally to this work.
}

Citation: Franz, M.

Wiciak-Pawłowska, K.; Franz, J.

Binary-Encounter Model for Direct

Ionization of Molecules by

Positron-Impact. Atoms 2021, 9, 99.

https://doi.org/10.3390/

atoms 9040099

Academic Editor: Grzegorz Piotr Karwasz

Received: 24 October 2021

Accepted: 20 November 2021

Published: 24 November 2021

Publisher's Note: MDPI stays neutral with regard to jurisdictional claims in published maps and institutional affiliations.

Copyright: (C) 2021 by the authors. Licensee MDPI, Basel, Switzerland. This article is an open access article distributed under the terms and conditions of the Creative Commons Attribution (CC BY) license (https:/ / creativecommons.org/licenses/by/ $4.0 /)$.

\begin{abstract}
We introduce two models for the computation of direct ionization cross sections by positron impact over a wide range of collision energies. The models are based on the binary-encounter-Bethe model and take into account an extension of the Wannier theory. The cross sections computed with these models show good agreement with experimental data. The extensions improve the agreement between theory and experiment for collision energies between the first ionization threshold and the peak of the cross section. The models are based on a small set of parameters, which can be computed with standard quantum chemistry program packages.
\end{abstract}

Keywords: positron impact ionization; positron-molecule scattering; binary-encounter-Bethe

\section{Introduction}

We present two new models for the computation of cross sections for direct ionization of atoms and molecules by positron-impact. Positrons can ionize atoms and molecules by direct annihilation, positronium (Ps) formation and direct ionization. It is difficult to distinguish these channels in scattering experiments. As a consequence, the recent review articles by Brunger et al. [1] and Ratnavelu et al. [2] show only the sum of the cross sections for Ps-formation and direct ionization for the majority of targets. This shows the need for the development of an accurate theoretical model for the computation of the cross sections for direct ionization. Such a model can be used to deduct the cross section for Ps-formation from cross sections that cannot distinguish between different ionization channels.

For electron-impact ionization, Kim and Rudd [3,4] developed the binary-encounterBethe (BEB) model. In this model, the binary-encounter version of the Mott cross section for hard collisions at low collision energies is joined with the Bethe model for soft collisions at high collisions energies. The model gives ionization cross sections for molecules that contain light atoms with an accuracy of 10 percent over an energy range from the ionization threshold up to a few thousand electron volts. Another advantage of the BEB model is that it requires only quantities, which can be computed with standard quantum chemistry computer program packages. An attractive feature of this model is that it is free of any fitting parameters.

In order to formulate a BEB model for positron impact ionization, the similarities and differences between electron and positron impact have to be taken into account. For high collision energies-above a few hundred eV - the cross sections for both projectiles are similar. For positron-impact ionization, there is no exchange interaction between the projectile and the target. For energies close to the ionization threshold, the cross sections for electron and positron impact ionization are expected to be different because of the different charges of the projectiles. In the case of electron impact ionization, two electrons are ejected in opposite directions because of electron-electron repulsion. In contrast, after ionization by positron impact, an electron and a positron move in the same direction because of the 
mutual attraction (see, e.g., Chapter 5 in Charlton and Humberston [5]). Klar [6] showed, using Wannier theory [7], that, just above the ionization threshold, the cross sections for electron-impact and positron impact follow a power law of the type

$$
\sigma(E) \propto(E-B)^{-\alpha} .
$$

Here, $E$ is the energy of the incoming projectile and $B$ is the binding energy of the ejected electron. The exponent $\alpha$ has the value 1.127 if the projectile is an electron and 2.651 if the projectile is a positron. Recently Fedus and Karwasz [8] derived a binary-encounterBethe (BEB) model for positron-impact ionization. Their model is very similar to the BEB model of Kim and Rudd [3] for electron-impact ionization, but without the term describing the electron-exchange interaction. Furthermore Fedus and Karwasz [8] take the Wannier threshold law into account to correct the cross section for collision energies close to the ionization threshold. This is discussed in more detail in Section 2.

Rost and Heller [9] derived a similar threshold law with the help of semi-classical Feynman path integrals and predicted that the Wannier-type threshold law for positron impact is valid for energies up to about $3 \mathrm{eV}$ above the ionization threshold. In order to increase the validity of the Wannier theory towards higher energies, Ihra et al. [10] extended it with unharmonic corrections. They derived a threshold law of the form

$$
\sigma(E) \propto(E-B)^{-\alpha} e^{-\beta \sqrt{(E-B)}},
$$

with the values $\alpha=2.640$ and $\beta=0.73$. This equation agrees very well for energies up to $10 \mathrm{eV}$ above threshold with the experimental data of Ashley et al. [11] for the cross section for positron-impact ionization of helium. More recently, Jansen et al. [12] extended this approach by including the contribution from higher partial waves, whereas Ihra et al. [10] considered only the lowest partial wave $(L=0)$, Jansen et al. [12] included the first 4 partial waves and found a large contribution from the $D$-wave. Their final expression for the threshold law is similar to the expression from Ihra et al. [10] but with the parameters $\alpha=2.640$ and $\beta=0.489$.

The purpose of this paper is to generalize the BEB-model for positrons [8] to follow the threshold law derived by Ihra et al. [10] with the parameters given in Jansen et al. [12]. In Section 2, we present the two theoretical models of Fedus and Karwasz [8], and two new models, which fulfill the threshold law of Jansen et al. [12]. In Section 3, we show comparisons between the theory and all recommended direct ionization cross section listed in the review by Brunger et al. [1]. In Section 4, the paper ends with conclusions.

\section{Theoretical Model}

The total cross section for direct ionization by positron impact can be written as the sum of the partial ionization cross sections for the $n_{o c c}$ occupied orbitals

$$
\sigma(E)=\sum_{i}^{n_{o c c}} \sigma_{i}(E)
$$

Here, $\sigma_{i}(E)$ is the partial ionization cross section for ionization from orbital $i$. Each of them will be computed with the BEB models described below.

\subsection{BEB-0 Model}

Following the BEB model of Fedus and Karwasz [8], the partial ionization cross section is given by

$$
\sigma_{i}^{\mathrm{BEB}}(E)=\frac{S_{i}}{E+U_{i}+B_{i}} \frac{B_{i}}{E}\left[\left(E-B_{i}\right)+\frac{1}{2 E}\left(E-B_{i}\right)\left(E+B_{i}\right) \ln \frac{E}{B_{i}}\right] .
$$


Here, $E$ is the kinetic energy of the incoming positron, $B_{i}$ is the electron binding energy in orbital $i$ and $U_{i}$ is the expectation value of the kinetic energy of the bound electron in orbital $i$. Here, all energies are given in $\mathrm{eV}$. The energy-independent prefactor is given by

$$
S_{i}=4 \pi a_{0}^{2} N_{i}\left(\frac{R}{B_{i}}\right)^{2}
$$

where $a_{0}=0.529 \times 10^{-10} \mathrm{~m}$ is the Bohr radius, $N_{i}$ is the occupation number of the orbital and $R=13.6 \mathrm{eV}$ is the Rydberg constant.

With the introduction of the reduced variables

$$
t_{i}=\frac{E}{B_{i}} \text { and } u_{i}=\frac{U_{i}}{B_{i}}
$$

the cross section can be written in the compact form, which we will refer to as BEB-0 model

$$
\sigma_{i}^{\mathrm{BEB}-0}=\frac{S_{i}}{t_{i}+u_{i}+1}\left[\frac{\ln t_{i}}{2}\left(1-\frac{1}{t_{i}^{2}}\right)+1-\frac{1}{t_{i}}\right] .
$$

\subsection{BEB-W Model}

Fedus and Karwasz [8] introduced a scaling function in the denominator of the term in front of the brackets on the right hand side of Equation (7). This term regulates the behavior of the cross section at energies closely above the ionization threshold and ensures that the cross section follows the Wannier law. In the following, we call this the BEB-W model. The partial cross section is given by

$$
\sigma_{i}^{\mathrm{BEB}-\mathrm{W}}=\frac{S_{i}}{t_{i}+u_{i}+1_{i}+f_{i}^{\mathrm{W}}}\left[\frac{\ln t_{i}}{2}\left(1-\frac{1}{t_{i}^{2}}\right)+1-\frac{1}{t_{i}}\right] .
$$

Here, the scaling function is given by

$$
f_{i}^{\mathrm{W}}=\frac{C}{\left(t_{i}-1\right)^{1.65}} .
$$

Here, the exponent 1.65 follows from the exponent in the Wannier theory of Klar [6]. The exact value of the constant $C$ is not known, and the Wannier law gives only the proportionality of the cross section. In practical application, a value of $C=1$ is chosen [8].

\subsection{BEB-A Model}

In a similar way, we can modify the BEB-0 model so that it fulfills the threshold law derived by Jansen et al. [12]. We define the following expression as the BEB-A model

$$
\sigma_{i}^{\mathrm{BEB}-\mathrm{A}}=\frac{S_{i}}{t_{i}+u_{i}+1+f_{i}^{\mathrm{A}}}\left[\frac{\ln t_{i}}{2}\left(1-\frac{1}{t_{i}^{2}}\right)+1-\frac{1}{t_{i}}\right] .
$$

Here, we introduced the scaling function

$$
f_{i}^{\mathrm{A}}=\frac{C^{\prime}}{\left(t_{i}-1\right)^{\alpha-1} e^{-\beta_{i} \sqrt{t_{i}-1}}},
$$

where $\alpha=2.640$. We choose the constant $C^{\prime}=1$; its value is not specified by the threshold law by Jansen et al. [12]. Here, we introduced the value

$$
\beta_{i}=0.489 \sqrt{\frac{B_{i}}{2 R}}
$$


which depends on the orbital $i$. This follows from the use of the reduced kinetic energy $t_{i}$ in the expression for the cross section, instead of the excess kinetic energy $\Delta E=E-B_{i}$ as in the formulation of the threshold law.

\subsection{BEB-B Model}

The BEB-0 model can be modified in different ways to fulfill the threshold law of Jansen et al. [12]. An alternative is the introduction of an additional term in the brackets on the right hand side of Equation (7). We suggest the following expression for the partial ionization cross section, which we will call BEB-B model.

$$
\sigma_{i}^{\mathrm{BEB}-\mathrm{B}}=\frac{S_{i}}{t_{i}+u_{i}+1}\left[\frac{\ln t_{i}}{2}\left(1-\frac{1}{t_{i}^{2}}\right)+h_{i}\left(t_{i}\right)\left(1-\frac{1}{t_{i}}\right)+g_{i}\left(t_{i}\right)\left(1-\frac{1}{t_{i}}\right)^{\alpha}\right] .
$$

Here, the prefactor in the additional term is given by

$$
g_{i}\left(t_{i}\right)=C_{i} e^{-\beta_{i} \sqrt{t_{i}-1}}
$$

where the coefficients $C_{i}$ are not specified by the threshold law, and we set them equal to 1. The product of the two terms is exactly the threshold law of Jansen et al. [12]. The exponential factor decreases for increasing energies and switches this term off for high energies. The function

$$
h_{i}\left(t_{i}\right)=1-g_{i}\left(t_{i}\right)
$$

switches on the second term in the brackets at collision energies, for which the cross section reaches its maximum value.

\section{Results and Discussion}

In the review by Brunger et al. [1], cross sections for direct ionization are recommended only for the four molecules: hydrogen $\left(\mathrm{H}_{2}\right)$, nitrogen $\left(\mathrm{N}_{2}\right)$, oxygen $\left(\mathrm{O}_{2}\right)$ and carbon monoxide $(\mathrm{CO})$. In the following, we present results for all four molecules.

\subsection{Molecular Hydrogen}

The review by Brunger et al. [1] recommends for the direct ionization of molecular hydrogen by positron impact the experimental cross section data from Fromme et al. [13]. In these experiments the authors did not distinguish between the channels for direct ionization and Ps-formation. More recent experiments by Jacobsen et al. [14] took great care to distinguish between these different channels. For energies up to $100 \mathrm{eV}$, their cross section is about 30 percent lower than the values from Fromme et al. [13]. Recent calculations by Utamuratov et al. [15] with the convergent close-coupling (CCC) method are closer to the data from Jacobsen et al. [14] than to the data from Fromme et al. [13]. Therefore, we compare the results from the various BEB models with the data from Jacobsen et al. [14].

In Figure 1, we compare the cross sections for direct ionization from hydrogen molecules. The figure shows the computed cross section, which we obtained with the four BEB models, the experimental data from Jacobsen et al. [14] and the calculations by Utamuratov et al. [15] with the CCC method. In our calculations, we used the molecular parameters listed in the Hwang et al. [4], which are the same as those used by Fedus and Karwasz [8]. Therefore curves for BEB-0 and BEB-W are identical to those by Fedus and Karwasz [8]. The BEB-0 model gives the largest cross sections. The second largest cross sections are computed by the BEB-W model, followed by the BEB-B and BEB-A models. Close to the threshold, the BEB-B model gives slightly larger cross sections than the BEB-W model. For collision energies above $200 \mathrm{eV}$, all four BEB models give nearly identical cross sections. All of them are slightly larger than the experimental cross sections. For collision energies below $100 \mathrm{eV}$, the BEB-0 and BEB-W models overestimate the cross section, whereas the BEB-A and BEB-B models are very close to the experimental points. The cross sections computed with the CCC method overestimate the experimental cross 
sections for collision energies up to $100 \mathrm{eV}$. Here, results from the CCC method are very close to those obtained with the BEB- 0 model. Between $100 \mathrm{eV}$ and $500 \mathrm{eV}$, the CCC-results are close to the experimental data. Above $500 \mathrm{eV}$, they are close to the results from the four BEB models and are higher than the experimental values.

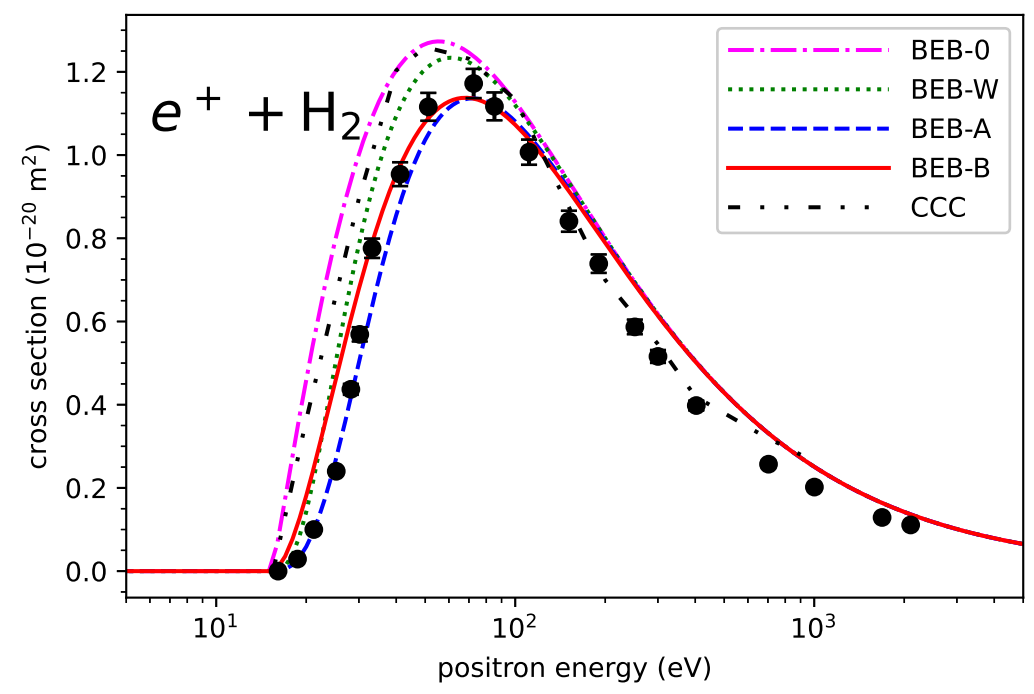

Figure 1. Direct ionization cross sections from $\mathrm{H}_{2}$ molecules by positron impact. The results from calculations with the four different BEB models are shown by the magenta dash-dotted line (BEB-0 model), the green dotted line (BEB-W model), the blue dashed line (BEB-A model) and the solid red line (BEB-B model). The data from the calculations by Utamuratov et al. [15] with the CCC method are shown by the black dash-dotted-dotted line. Also shown are the experimental data (solid black circles with error-bars) from Jacobsen et al. [14]. The error-bars correspond to the experimental uncertainties of 3 per cent given by Jacobsen et al. [14].

\subsection{Molecular Nitrogen}

In Figure 2, we show the cross sections for direct ionization of molecular nitrogen by positron impact computed with the four BEB models. Also shown is the experimental data set from Marler and Surko [16], which is recommended in the review by Brunger et al. [1]. In the calculations with the BEB models, we used the molecular parameters listed in the Hwang et al. [4], which are also used by Fedus and Karwasz [8]. The BEB-0 model gives the largest cross sections, followed by the BEB-W model. The BEB-A and BEB-B model give nearly the same cross sections over the whole energy range. For collision energies above $200 \mathrm{eV}$, all four BEB-models give very similar cross sections. For collision energies below $100 \mathrm{eV}$, the BEB-0 and BEB-W models overestimate the cross section, whereas the BEB-A and BEB-B models are very close to the experimental values.

\subsection{Molecular Oxygen}

In Figure 3, we show the cross sections for direct ionization of molecular oxygen by positron impact computed with the various BEB models. As for molecular nitrogen, we show in the same figure the experimental data set from Marler and Surko [16], which is recommended in the review by Brunger et al. [1]. In the calculations with the BEB models, we used the molecular parameters given in the Hwang et al. [4]. These same values for these parameters are used by Fedus and Karwasz [8]. As observed for hydrogen and nitrogen, the BEB-0 model gives the largest cross sections, followed by the BEB-W model, followed by the BEB-A and BEB-B models, which give very similar results. For collision energies above $200 \mathrm{eV}$, the cross sections obtained with the four BEB-models are nearly identical. For collision energies below $100 \mathrm{eV}$, all four models are close to the experimental values and within the experimental uncertainties. 


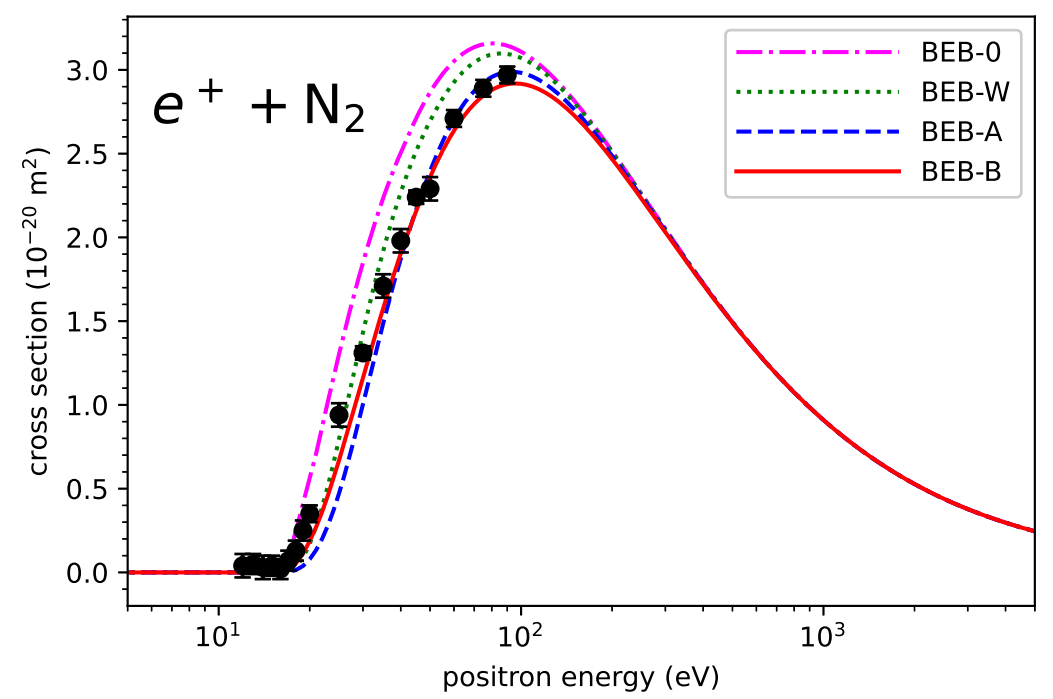

Figure 2. Direct ionization cross sections from $\mathrm{N}_{2}$ molecules by positron impact. The results from calculations with the four different BEB models are shown by the magenta dash-dotted line (BEB-0 model), the green dotted line (BEB-W model), the blue dashed line (BEB-A model) and the solid red line (BEB-B model). Also shown are the experimental data (solid black circles with error-bars) from Marler and Surko [16]. The values of the error-bars show the experimental uncertainties are taken from the Table 12 in the review by Brunger et al. [1].

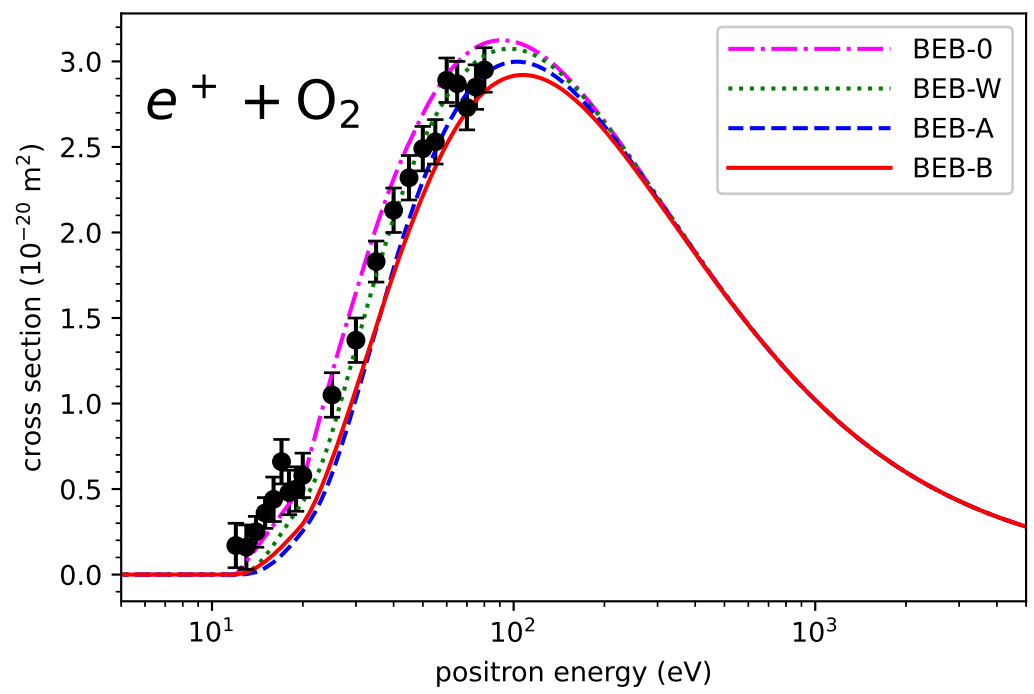

Figure 3. Direct ionization cross sections from $\mathrm{O}_{2}$ molecules by positron impact. The results from calculations with the four different BEB models are shown by the magenta dash-dotted line (BEB-0 model), the green dotted line (BEB-W model), the blue dashed line (BEB-A model) and the solid red line (BEB-B model). Also shown are the experimental data (solid black circles with error-bars) from Marler and Surko [16]. The values of the error-bars show the experimental uncertainties are taken from the Table 15 in the review by Brunger et al. [1].

\subsection{Carbon Monoxide}

In Figure 4, we show the cross sections for direct ionization of carbon monoxide by positron impact. The results from calculations with the four different BEB models are shown together with the experimental data set from Marler and Surko [16], which is again the recommended data set in the review by Brunger et al. [1]. As already observed for the other targets, the BEB-0 model gives the largest cross section, followed by the BEB-W model. The cross sections computed with the BEB-A and BEB-B models are the smallest ones in this set and are very similar to each other. Above $200 \mathrm{eV}$, all four models give nearly 
identical results. The experimental data from Marler and Surko [16] are only available from the ionization threshold up to about $100 \mathrm{eV}$. For this target, the BEB- 0 model agrees best with the available experimental data. The reason for the inferior performance of the BEB-A and BEB-B models might be due to the dipole moment of the carbon monoxide molecule. The threshold laws of Jansen et al. [12] have been derived for the ionization of atoms that are non-polar species. However, one should also keep in mind that there is a larger spread among the experimental data points than the other targets, and no experimental uncertainties are available for this set of data in Marler and Surko [16] or in the review by Brunger et al. [1].

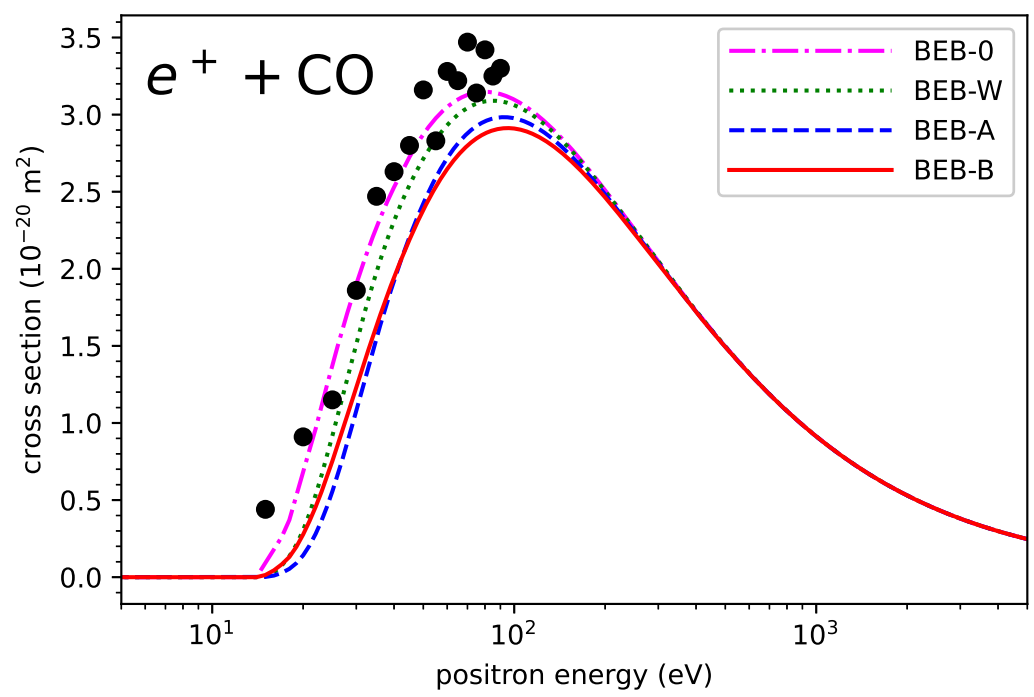

Figure 4. Direct ionization cross sections from $\mathrm{CO}$ molecules by positron impact. The results from calculations with the four different BEB models are shown by the magenta dash-dotted line (BEB-0 model), the green dotted line (BEB-W model), the blue dashed line (BEB-A model) and the solid red line (BEB-B model). Also shown are the experimental data (solid black circles) from Marler and Surko [16].

\section{Conclusions}

We introduced two binary-encounter Bethe models (BEB-A and BEB-B) for the calculation of cross sections for direct ionization of molecules by positron impact. Both models fulfill the threshold laws, derived by Jansen et al. [12]. We compared these models with the BEB-0 and BEB-W models from Fedus and Karwasz [8]. In the energy range from ionization threshold up to the maximum of the cross section around $100 \mathrm{eV}$, the BEB-A and BEB-B models show better agreement with the available experimental data for molecular hydrogen and nitrogen. For molecular oxygen, the cross sections computed with all four BEB models are within the experimental uncertainties. Carbon monoxide is the only polar molecule in the test set. Here, the BEB-0 model performs best, followed by the BEB-W model. This suggests that the influence of the dipole moment on the ionization cross section is important for energies from the ionization threshold up to the peak of the cross section.

Only a very limited number of cross sections for direct ionization by positron impact have been measured [1,2]. Furthermore, ab initio calculations are only available for the hydrogen molecule [15]. It is not expected that this situation will change in the next few years, because of the difficulty to distinguish direct ionization and Ps-formation channels experimentally. Taking into account the limitations, our present conclusions are: the BEBA and BEB-B models are the best models for the calculation of cross sections for direct ionization by positron impact from non-polar molecules, and for polar molecules, the BEB-0 model seems to be the most reliable method.

There are more experimental data available for total ionization cross sections, which are the sums of cross sections for direct ionization and Ps-formation [1]. The BEB models 
discussed in this paper can be useful to extract the cross section for Ps-formation from this data.

Recently we suggested [17] to build up a database with cross sections for positron collision with molecules of biological interest. In the past few years, we computed elastic cross sections for various biomolecules [18-21]. The BEB model from this paper will enable us to fill the database with more data.

In two other recent publications [22,23], we computed elastic cross sections with the R-matrix method [24] and substracted them from the experimental total cross sections [1]. With this procedure, we obtained the sum of the cross sections for Ps-formation, direct ionization and other inelastic processes (e.g., electronic excitation). The BEB-model from this paper can help to disentangle the data further. Such cross section data are important for simulations of the effects of ion-beams on biological materials that are currently limited to secondary electrons (see, e.g., Taioli et al. [25]).

Author Contributions: Conceptualization, M.F. and J.F.; methodology, M.F., K.W.-P. and J.F.; software, M.F., K.W.-P. and J.F.; validation, M.F., K.W.-P. and J.F.; formal analysis, M.F., K.W.-P. and J.F.; investigation, M.F., K.W.-P. and J.F.; resources, M.F.; data curation, J.F.; writing-original draft preparation, M.F., K.W.-P. and J.F.; writing—review and editing, M.F.; visualization, M.F., K.W.-P. and J.F.; supervision, J.F.; project administration, J.F.; funding acquisition, J.F. All authors have read and agreed to the published version of the manuscript.

Funding: The research has been supported by the computer centres WCSS (Wroclawskie Centrum Sieciowo-Superkomputerowe, Politechnika Wroclawska) through grant number KDM-408 and CI TASK (Centrum Informatyczne Trójmiejskiej Akademickiej Sieci Komputerowej, Politechnika Gdańska).

Institutional Review Board Statement: Not applicable.

Informed Consent Statement: Not applicable.

Data Availability Statement: The data that support the findings of this study will be soon openly available in the MOST Wiedzy repository (https://mostwiedzy.pl/en/open-research-data/catalog, accessed on 22 November 2021).

Conflicts of Interest: The authors declare no conflict of interest.

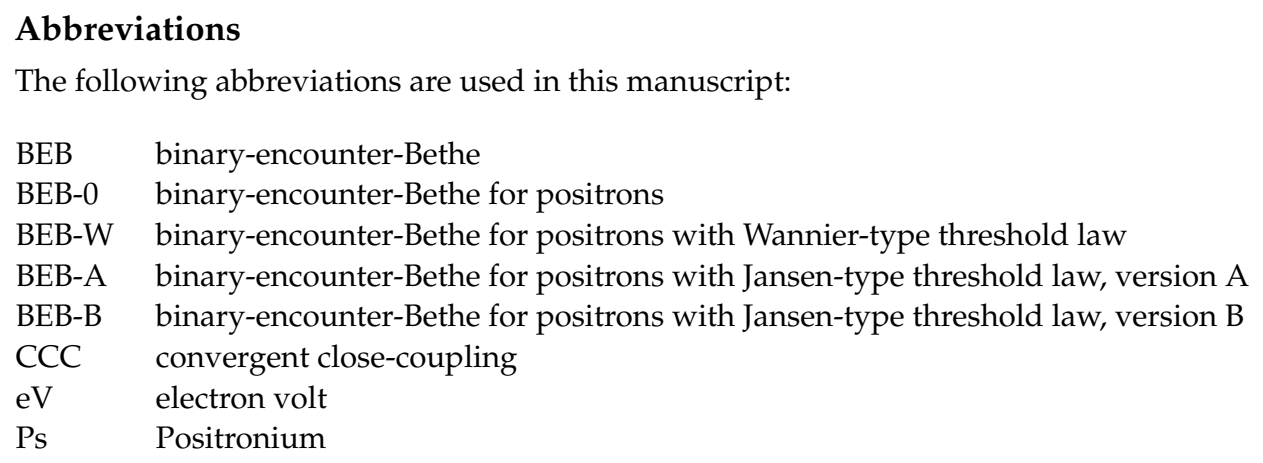

\section{References}

1. Brunger, M.; Buckman, S.J.; Ratnavelu, K. Recommended Positron Scattering Cross Sections for Atomic Systems. Positron Scattering from Molecules: An Experimental Cross Section Compilation for Positron Transport Studies and Benchmarking Theory. J. Phys. Chem. Ref. Data 2017, 46, 023102. [CrossRef]

2. Ratnavelu, K.; Brunger, M.J.; Buckman, S.J. Recommended Positron Scattering Cross Sections for Atomic Systems. J. Phys. Chem. Ref. Data 2019, 48, 023102. [CrossRef]

3. Kim, Y.-K.; Rudd, M.E. Binary-encounter-dipole model for electron-impact ionization. Phys. Rev. A 1994, 50, 3954-3967. [CrossRef] [PubMed]

4. Hwang, W.; Kim, Y.-K.; Rudd, M.E. New model for electron-impact ionization cross sections of molecules. J. Phys. Chem. 1995, 104, 2956-2966. [CrossRef]

5. Charlton, M.; Humberston, J.W. Positron Physics; Cambridge University Press: Cambridge, UK, 2001.

6. Klar, H. Threshold ionisation of atoms by positrons. J. Phys. B At. Mol. Opt. Phys. 1981 14, 4165-4170. [CrossRef] 
7. Wannier, G.H. The Threshold Law for Single Ionization of Atoms or Ions by Electrons. Phys. Rev. 1953, 90, 817-825. [CrossRef]

8. Fedus, K.; Karwasz, G.P. Binary-encounter dipole model for positron-impact direct ionization. Phys. Rev. A 2019, 100, 062702. [CrossRef]

9. Rost, J.M.; Heller, E.J. Ionization of hydrogen by positron impact near the fragmentation threshold. Phys. Rev. A 1994, 49, R4289-R4292. [CrossRef]

10. Ihra, W.; Macek, J.H.; Mota-Furtado, F.; O'Mahony, P.F. Threshold Law For Positron Impact Ionization of Atoms. Phys. Rev. Lett. 1997, 78, 4027-4030. [CrossRef]

11. Ashley, P.; Moxom, J.; Laricchia, G. Near-Threshold Ionization of He and $\mathrm{H}_{2}$ by Positron Impact. Phys. Rev. Lett. 1996, 77, 1250-1253. [CrossRef]

12. Jansen, K.; Ward, S.J.; Shertzer, J.; Macek, J.H. Absolute cross sections for positron impact ionization of hydrogen near threshold. Phys. Rev. A 2009, 79, 022704. [CrossRef]

13. Fromme, D.; Kruse, G.; Raith, W.; Sinapius, G. Ionisation of molecular hydrogen by positrons. J. Phys. B At. Mol. Opt. Phys. 1988, 21, L261. [CrossRef]

14. Jacobsen, F.M.; Frandsen, N.P.; Knudsen, H.; Mikkelsen, U. Non-dissociative single ionization of molecular hydrogen by electron and positron impact. J. Phys. B At. Mol. Opt. Phys. 1995, 28, 4675-4689. [CrossRef]

15. Utamuratov, R.; Kadyrov, A.S.; Fursa, D.V.; Zammit, M.C.; Bray, I. Two-center close-coupling calculations of positron-molecularhydrogen scattering. Phys. Rev. A 2015, 92, 032707. [CrossRef]

16. Marler, J.P.; Surko, C.M. Positron-impact ionization, positronium formation, and electronic excitation cross sections for diatomic molecules. Phys. Rev. A 2005, 72, 062713. [CrossRef]

17. Franz, J. The POCOBIO Database for Computed Scattering Cross-Sections for Positron Collisions with Biomolecular Systems. Acta Phys. Pol. A 2017, 132, 1478-1481. [CrossRef]

18. Franz, J.; Gianturco, F.A. Low-energy positron scattering from gas-phase tetrahydrofuran: A quantum treatment of the dynamics and a comparison with experiments. J. Chem. Phys. 2013, 139, 204309. [CrossRef] [PubMed]

19. Franz, J.; Gianturco, F.A. Low-energy positron scattering from gas-phase pyrimidine: A quantum treatment of the dynamics and a comparison with experiments. Phys. Rev. A 2013, 88, 042711. [CrossRef]

20. Franz, J.; Gianturco, F.A.; Baccarelli, I. Low-energy positron scattering from gas-phase uracil. Eur. Phys. J. D 2014, 68, 183. [CrossRef]

21. Franz, J.; Gianturco, F.A. Low-energy positron scattering from DNA nucleobases: The effects from permanent dipoles. Eur. Phys. J. D 2014, 68, 279. [CrossRef]

22. Franz, J.; Franz, M. Low-energy positron scattering from gas-phase benzene. Eur. Phys. J. D 2019, 73, 192. [CrossRef]

23. Karbowski, A.; Karwasz, G.P.; Franz, M.; Franz, J. Positron Scattering and Annihilation in Organic Molecules. Acta Phys. Pol. B 2020, 51, 207-212. [CrossRef]

24. Baluja, K.; Zhang, R.; Franz, J.; Tennyson, J. Low-energy positron collisions with water: Elastic and rotationally inelastic scattering. J. Phys. B At. Mol. Opt. Phys. 2007, 40, 3515-3524. [CrossRef]

25. Taioli, S.; Trevisanutto, P.E.; de Vera, P.; Simonucci, S.; Abril, I.; Garcia-Molina, R.; Dapor, M. Relative Role of Physical Mechanisms on Complex Biodamage Induced by Carbon Irradiation. J. Phys. Chem. Lett. 2021, 12, 487-493. [CrossRef] [PubMed] 